\title{
A CHARACTERIZATION OF REGULAR SADDLE SURFACES IN THE HYPERBOLIC AND SPHERICAL THREE-SPACE
}

\author{
DIMITRIOS E. KALIKAKIS
}

Received 7 March 2002

We prove that the class of regular saddle surfaces in the hyperbolic or spherical three-space coincides with the class of regular surfaces with curvature not greater than the curvature of the surrounding space. We also show that a similar result for nonregular surfaces is incorrect.

\section{Introduction}

Among surfaces in a three-dimensional Euclidean space $\mathbb{E}^{3}$, two classes of surfaces are of fundamental importance: the convex and saddle surfaces. A saddle surface is a surface from which it is impossible to cut off a crust by any plane. In the regular case, each of the above classes is closely connected with the sign of the Gaussian curvature; a regular convex surface has nonnegative Gaussian curvature at each point and a saddle surface has a nonpositive Gaussian curvature. In fact, the class of regular saddle surfaces in $\mathbb{E}^{3}$ coincides with the class of regular surfaces of nonpositive Gaussian curvature [3]. In our work, we extend the characterization of regular saddle surfaces in $\mathbb{E}^{3}$ to simply connected 3-manifolds of constant curvature. Notice that it is still an open question whether a regular surface in $\mathbb{E}^{n}(n>3)$ of nonpositive curvature is a saddle surface [1, page 12]. Our main result is the following theorem.

THeOREm 1.1. A regular surface in a hyperbolic or spherical three-space is a saddle surface if and only if its Gaussian curvature is everywhere not greater than the curvature of the surrounding space.

Theorem 1.1 and its proof employ a new metric definition of a saddle surface that is equivalent to the classical definition. This definition enables us to take advantage of the geodesic structure of spaces of constant curvature, as well as the existence of geodesic mappings between the Euclidean space and the hyperbolic 
and spherical three-space. For our purposes, we use the Beltrami-Klein model for the hyperbolic three-space because of its simple geodesic structure.

In Section 2, we briefly discuss saddle surfaces in spaces of constant curvature and their relation with geodesic mappings (Corollary 2.3). In Section 3, we prove Theorem 1.1, and in Section 4, we present an example showing that the condition of regularity in Theorem 1.1 is essential. Now, we discuss some necessary terminology.

The Beltrami-Klein model for the hyperbolic three-space $\mathbb{H}_{\kappa}^{3}(\kappa<0)$ is represented by the set $\left\{\left(x_{1}, x_{2}, x_{3}\right) \in \mathbb{R}^{3}: x_{1}^{2}+x_{2}^{2}+x_{3}^{2}<1 /(-\kappa)\right\}$ equipped with the metric tensor

$$
d s^{2}=\frac{\left(1 / \kappa+\sum_{i=1}^{3} x_{i}^{2}\right)\left(\sum_{i=1}^{3} d x_{i}^{2}\right)-\left(\sum_{i=1}^{3} x_{i} d x_{i}\right)^{2}}{\kappa\left(1 / \kappa+\sum_{i=1}^{3} x_{i}^{2}\right)^{2}} .
$$

In this model, a geodesic segment is a Euclidean line segment, and an orthogonal transformation of $\mathbb{R}^{3}$ is a Beltrami-Klein isometry (the converse is not correct). A regular surface in the Beltrami-Klein model of $\mathbb{H}_{\kappa}^{3}(\kappa<0)$ is a surface which can be locally represented as the graph of a $C^{2}$-function of two variables, which has everywhere a (nonzero) normal vector, and is inside the ball $x_{1}^{2}+x_{2}^{2}+x_{3}^{2}<$ $1 /(-\kappa)$.

The spherical three-space is an open hemisphere of $\mathbb{E}^{4}$,

$$
\mathbb{S}_{+}^{3}\left(\kappa^{-1 / 2}\right)=\left\{\left(x_{1}, x_{2}, x_{3}, x_{4}\right) \in \mathbb{R}^{4}: x_{1}^{2}+x_{2}^{2}+x_{3}^{2}+x_{4}^{2}=\frac{1}{\kappa}, x_{4}>0\right\}, \quad \kappa>0,
$$

equipped with the induced metric. As it is well known, a geodesic segment in this space is an arc of a great circle. A linear transformation of $\mathbb{R}^{4}$ represented by a matrix of the form $\left(\begin{array}{cc}A_{3 \times 3} & 0 \\ 0 & 1\end{array}\right)$ with $A$ orthogonal is an isometry of the upper open hemisphere in $\mathbb{E}^{4}$. A regular surface in $\mathbb{S}_{+}^{3}\left(\kappa^{-1 / 2}\right)$ is a surface represented in the form

$$
\mathbf{r}(x, y)=a\left(x, y, f(x, y), \kappa^{-1 / 2}\right),
$$

where $a=\left(1+\kappa x^{2}+\kappa y^{2}+\kappa f^{2}(x, y)\right)^{-1 / 2}$ and $f$ is a regular function of two variables.

Both $\mathbb{H}_{\mathcal{K}}^{3}$ and $\mathbb{S}_{+}^{3}\left(\kappa^{-1 / 2}\right)$ are Riemannian simply connected manifolds of constant sectional curvature $\kappa$ in which any pair of points can be joined by a unique geodesic segment.

\section{Saddle surfaces in $\mathbb{E}^{3}, \mathbb{H}_{\kappa}^{3}, \mathbb{S}_{+}^{3}\left(\kappa^{-1 / 2}\right)$}

In this section, $M$ denotes anyone of the spaces $\mathbb{E}^{3}, \mathbb{H}_{\kappa}^{3}, \mathbb{S}_{+}^{3}\left(\kappa^{-1 / 2}\right)$. The convex hull of a subset $A \subset M$, denoted by $\operatorname{conv}(A)$, is defined to be the smallest convex set which contains the subset $A$; $\operatorname{conv}(A)$ can be also realized as the union of 
all sets $G^{(n)}(A)$, with $G^{(0)}(A)=A, G^{(1)}(A)$ is the union of all geodesic segments between points of $A$, and $G^{(n)}(A)=G^{(1)}\left(G^{(n-1)}(A)\right)$ for any $n>1$.

A surface $f$ in $M$ is any continuous mapping $f: D \rightarrow M$, where $D$ denotes the closed unit disk on the plane. In the Euclidean space $\mathbb{E}^{3}$ we say that a plane $P$ cuts off a crust from the surface $f$ if among the connected components of $f^{-1}(f(D) \backslash P)$ there is one with positive distance from the boundary of $D$. If $U$ is such a component, then the set $f(U)$ is called a crust.

A surface $f$ in $\mathbb{E}^{3}$ is said to be a saddle surface if it is impossible to cut off a crust from it by any plane.

Saddle surfaces in $\mathbb{H}_{\mathcal{K}}^{3}$ and $\mathbb{S}_{+}^{3}\left(\kappa^{-1 / 2}\right)$ can be defined by means of the operation of cutting off crusts by hyperbolic or spherical planes. Instead of this definition we will make use of the following equivalent coordinate free definition, first introduced in [2], which depends only on the geodesic structure of the surrounding space.

Definition 2.1. A surface $f$ in $\mathbb{H}_{\kappa}^{3}$ and $\mathbb{S}_{+}^{3}\left(\kappa^{-1 / 2}\right)$ is said to be a saddle surface if

$$
f(\text { int } \gamma) \subset \operatorname{conv}(f(\gamma))
$$

for every Jordan curve $\gamma$ in the closed unit disk having positive distance from the unit circle.

Saddle surfaces in $\mathbb{H}_{\kappa}^{3}$ and $\mathbb{S}_{+}^{3}\left(\kappa^{-1 / 2}\right)$ can be simply characterized (Corollary 2.3) by means of the following two geodesic mappings between the Euclidean space and $\mathbb{H}_{\mathcal{\kappa}}^{3}$ and $\mathbb{S}_{+}^{3}\left(\kappa^{-1 / 2}\right)$. A mapping between two spaces which preserves geodesic segments is called a geodesic mapping.

The inclusion mapping id : $\mathbb{H}_{\kappa}^{3} \rightarrow \mathbb{E}^{3}$. Consider the Beltrami-Klein model of $\mathbb{H}_{\kappa}^{3}$ $(\kappa<0)$. Since geodesic segments in the Beltrami-Klein model of $\mathbb{H}_{\kappa}^{3}$ coincide with the Euclidean line segments, the inclusion mapping id : $\mathbb{H}_{\mathcal{K}}^{3} \rightarrow \mathbb{E}^{3}$ with $\operatorname{id}(x)=x$ and its inverse are geodesic mappings.

The central projection $\varphi: \mathbb{S}_{+}^{3}\left(\kappa^{-1 / 2}\right) \rightarrow \mathbb{E}^{3}$. Consider the central projection $\varphi: \mathbb{S}_{+}^{3}\left(\kappa^{-1 / 2}\right) \rightarrow \mathbb{E}^{3}(\kappa>0)$ with $\varphi\left(x_{1}, x_{2}, x_{3}, x_{4}\right)=\kappa^{-1 / 2}\left(x_{1} / x_{4}, x_{2} / x_{4}, x_{3} / x_{4}\right)$. The central projection takes a point $x$ on $\mathbb{S}_{+}^{3}\left(\kappa^{-1 / 2}\right)$ to the intersection of the hyperplane $\left\{x_{4}=\mathcal{\kappa}^{-1 / 2}\right\} \equiv \mathbb{E}^{3}$ with the straightline through the point $x$ and the origin of $\mathbb{E}^{4}$. Under the mapping $\varphi$ great circles go to straightlines and vice versa. Therefore, both $\varphi$ and $\varphi^{-1}$ are geodesic mappings.

Let $N$ be anyone of the spaces $\mathbb{E}^{3}, \mathbb{H}_{\kappa}^{3}, \mathbb{S}_{+}^{3}\left(\mathcal{\kappa}^{-1 / 2}\right)$. The next proposition follows directly from the definition of saddle surfaces and convex hull.

Proposition 2.2. If $f: D \rightarrow M$ is a saddle surface, and $\varphi: M \rightarrow N$ is a continuous geodesic mapping, then $\varphi \circ f$ is a saddle surface in $N$.

Corollary 2.3. (i) A surface in $\mathbb{H}_{\kappa}^{3}$ is a saddle surface if and only if it is a saddle surface in $\mathbb{E}^{3}$. (ii) A surface in $\mathbb{S}_{+}^{3}\left(\kappa^{-1 / 2}\right)$ is a saddle surface if and only if its image under the central projection is a saddle surface in $\mathbb{E}^{3}$. 


\section{Proof of Theorem 1.1}

The hyperbolic space. Let $\mathscr{S}$ be a regular surface in the Beltrami-Klein model of $\mathbb{H}_{\kappa}^{3}$ represented as the graph of a function $z=f(x, y)$ in a neighborhood of a point $\mathfrak{p}=(a, b, f(a, b))$.

Assume that $f_{x}(a, b)=f_{y}(a, b)=0$. A parametrization of the surface $\mathscr{S}$ is given by the vector-valued function $\mathbf{r}(x, y)=(x, y, f(x, y))$. The coefficients of the first fundamental form are given by

$$
\begin{aligned}
& E=\left\langle\mathbf{r}_{x}, \mathbf{r}_{x}\right\rangle=g_{11}+2 g_{13} f_{x}+g_{33} f_{x}^{2}, \\
& F=\left\langle\mathbf{r}_{x}, \mathbf{r}_{y}\right\rangle=g_{12}+g_{23} f_{x}+g_{13} f_{y}+g_{33} f_{x} f_{y}, \\
& G=\left\langle\mathbf{r}_{y}, \mathbf{r}_{y}\right\rangle=g_{22}+2 g_{23} f_{y}+g_{33} f_{y}^{2},
\end{aligned}
$$

where

$$
\begin{gathered}
g_{11}=\frac{1 / \kappa+y^{2}+z^{2}}{\kappa\left(1 / \kappa+x^{2}+y^{2}+z^{2}\right)^{2}}, \quad g_{22}=\frac{1 / \kappa+x^{2}+z^{2}}{\kappa\left(1 / \kappa+x^{2}+y^{2}+z^{2}\right)^{2}}, \\
g_{33}=\frac{1 / \kappa+x^{2}+y^{2}}{\kappa\left(1 / \kappa+x^{2}+y^{2}+z^{2}\right)^{2}}, \quad g_{12}=g_{21}=\frac{-x y}{\kappa\left(1 / \kappa+x^{2}+y^{2}+z^{2}\right)^{2}}, \\
g_{13}=g_{31}=\frac{-x z}{\kappa\left(1 / \kappa+x^{2}+y^{2}+z^{2}\right)^{2}}, \quad g_{23}=g_{32}=\frac{-y z}{\kappa\left(1 / \kappa+x^{2}+y^{2}+z^{2}\right)^{2}} .
\end{gathered}
$$

The Gaussian curvature can be expressed only in terms of $E, F, G$. A straightforward calculation implies that the hyperbolic Gaussian curvature $\kappa_{\mathbb{H}_{k}^{3}}(x, y$, $f(x, y))$ of the surface $\mathscr{S}$ at the point $(x, y, f(x, y))$, in the case when $f_{x}=f_{y}=0$, is equal to

$$
\frac{\left(1+\kappa x^{2}+\kappa y^{2}+\kappa f^{2}\right)^{2}\left(f_{x x} f_{y y}-f_{x y}^{2}\right)+\kappa\left(1+\kappa f^{2}\right)^{2}}{\left(1+\kappa f^{2}\right)^{2}} .
$$

Therefore,

$$
\kappa_{\mathbb{H}_{\kappa}^{3}}(a, b, f(a, b))=\frac{\left(1+\kappa a^{2}+\kappa b^{2}+\kappa f^{2}(a, b)\right)^{2}}{\left(1+\kappa f^{2}(a, b)\right)^{2}} \kappa_{\mathbb{E}^{3}}(a, b, f(a, b))+\kappa .
$$

Let $f_{x}(a, b) \neq 0$ or $f_{y}(a, b) \neq 0$, then consider the $3 \times 3$ matrix $Q$ defined by

$$
Q=\left[\begin{array}{ccc}
q_{11} & 0 & q_{13} \\
q_{21} & q_{22} & q_{23} \\
q_{31} & q_{32} & q_{33}
\end{array}\right]
$$

where $q_{11}=1 / \sqrt{1+f_{x}^{2}}, q_{13}=f_{x} / \sqrt{1+f_{x}^{2}}, q_{21}=-f_{x} f_{y} / \sqrt{1+2 f_{x}^{2}+f_{y}^{2}+f_{x}^{4}+f_{x}^{2} f_{y}^{2}}$, 
$q_{22}=\left(1+f_{x}^{2}\right) / \sqrt{1+2 f_{x}^{2}+f_{y}^{2}+f_{x}^{4}+f_{x}^{2} f_{y}^{2}}, \quad q_{23}=f_{y} / \sqrt{1+2 f_{x}^{2}+f_{y}^{2}+f_{x}^{4}+f_{x}^{2} f_{y}^{2}}$, $q_{31}=-f_{x} / \sqrt{1+f_{x}^{2}+f_{y}^{2}}, \quad q_{32}=-f_{y} / \sqrt{1+f_{x}^{2}+f_{y}^{2}}$, and $q_{33}=1 / \sqrt{1+f_{x}^{2}+f_{y}^{2}}$, where all partial derivatives are evaluated at the point $(a, b)$. The matrix $Q$ defines an orthogonal transformation by means of multiplication by column vectors, which transforms the normal vector $\mathbf{n}=\alpha\left(-f_{x}(a, b),-f_{y}(a, b), 1\right)$, where $\alpha=\left(1+f_{x}^{2}(a, b)+f_{y}^{2}(a, b)\right)^{-1 / 2}$, of the surface $\mathscr{S}$ at the point $\mathfrak{p}=(a, b, f(a, b))$ to the vector $(0,0,1)$. Let $\mathscr{S}^{\prime}$ be the image of the surface $\mathscr{S}$ under the action of $Q$, and $\mathfrak{p}^{\prime}$ the image of the point $\mathfrak{p}=(a, b, f(a, b))$. Since $Q$ preserves the inner product, the image of $\mathbf{n}$ (i.e., the vector $(0,0,1))$ is a normal vector to the surface $\mathscr{S}^{\prime}$ at the point $\mathfrak{p}^{\prime}$. This yields that in a neighborhood of $\mathfrak{p}^{\prime}$, the surface $\mathscr{S}^{\prime}$ can be represented as the graph of a new function $z=F(x, y)$. Hence, $\mathfrak{p}^{\prime}=\left(a^{\prime}, b^{\prime}, F\left(a^{\prime}, b^{\prime}\right)\right)$ and $\left(-F_{x}\left(a^{\prime}, b^{\prime}\right),-F_{y}\left(a^{\prime}, b^{\prime}\right), 1\right)=\beta(0,0,1)$, where $\beta=\sqrt{1+F_{x}^{2}\left(a^{\prime}, b^{\prime}\right)+F_{y}^{2}\left(a^{\prime}, b^{\prime}\right)}$. Therefore, $F_{x}\left(a^{\prime}, b^{\prime}\right)=F_{y}\left(a^{\prime}, b^{\prime}\right)=0$ and hence, by (3.4),

$$
\kappa_{\mathbb{H}_{\kappa}^{3}}\left(a^{\prime}, b^{\prime}, F\left(a^{\prime}, b^{\prime}\right)\right)=\frac{\left(1+\kappa a^{\prime 2}+\kappa b^{\prime 2}+\kappa F^{2}\left(a^{\prime}, b^{\prime}\right)\right)^{2}}{\left(1+\kappa F^{2}\left(a^{\prime}, b^{\prime}\right)\right)^{2}} \kappa_{\mathbb{E}^{3}}\left(a^{\prime}, b^{\prime}, F\left(a^{\prime}, b^{\prime}\right)\right)+\kappa .
$$

Being an orthogonal transformation, $Q$ is both a Euclidean and a BeltramiKlein isometry. By the theorema egregium, both the Euclidean and hyperbolic Gaussian curvatures of $\mathscr{Y}^{\prime}$ at $\mathfrak{p}^{\prime}$ are the same as the Euclidean and hyperbolic Gaussian curvatures of $\mathscr{S}$ at $\mathfrak{p}$, respectively. Thus, for any $a, b$ there are $a^{\prime}, b^{\prime}$ such that

$$
\kappa_{\mathbb{H}_{\kappa}^{3}}(a, b, f(a, b))=\frac{\left(1+\kappa a^{\prime 2}+\kappa b^{\prime 2}+\kappa F^{2}\left(a^{\prime}, b^{\prime}\right)\right)^{2}}{\left(1+\kappa F^{2}\left(a^{\prime}, b^{\prime}\right)\right)^{2}} \kappa_{\mathbb{E}^{3}}(a, b, f(a, b))+\kappa .
$$

Therefore, the hyperbolic curvature of a regular surface in $\mathbb{H}_{\kappa}^{3}$ is not greater than $\kappa$ if and only if its Euclidean curvature is nonpositive, or equivalently, if and only if the surface is a saddle surface in $\mathbb{E}^{3}$. Corollary 2.3(i) completes the proof of Theorem 1.1 in the hyperbolic case.

The spherical space. Let $\mathscr{S}$ be a regular surface in $\mathbb{S}_{+}^{3}\left(\kappa^{-1 / 2}\right)$ represented by an equation of the form (1.3) in a neighborhood of a point $\mathbf{r}(a, b)$. The coefficients of the first fundamental form are given by $E=\left\langle\mathbf{r}_{x}, \mathbf{r}_{x}\right\rangle, F=\left\langle\mathbf{r}_{x}, \mathbf{r}_{y}\right\rangle, G=$ $\left\langle\mathbf{r}_{y}, \mathbf{r}_{y}\right\rangle$, where $\langle\cdot, \cdot \cdot\rangle$ denotes the Euclidean inner product of $\mathbb{E}^{4}$. The Gaussian curvature can be expressed only in terms of $E, F, G$. Straightforward calculations yield that the Gaussian curvature of the surface $\mathscr{S}$ at the point $\mathbf{r}(x, y)$, in the case when $f_{x}=f_{y}=0$, is equal to $\left(\left(1+\kappa x^{2}+\kappa y^{2}+\kappa f^{2}\right)^{2}\left(f_{x x} f_{y y}-f_{x y}^{2}\right)+\right.$ $\left.\kappa\left(1+\kappa f^{2}\right)^{2}\right) /\left(1+\kappa f^{2}\right)^{2}$. Therefore,

$$
\kappa_{\mathbb{S}_{+}^{3}\left(\kappa^{-1 / 2}\right)}(\mathbf{r}(a, b))=\frac{\left(1+\kappa a^{2}+\kappa b^{2}+\kappa f^{2}(a, b)\right)^{2}}{\left(1+\kappa f^{2}(a, b)\right)^{2}} \kappa_{\mathbb{E}^{3}}(a, b, f(a, b))+\kappa .
$$


If $f_{x}(a, b) \neq 0$ or $f_{y}(a, b) \neq 0$, then we apply to the surface $\mathscr{S}$ an orthogonal transformation of the form $Q_{4 \times 4}=\left(\begin{array}{cc}A_{3 \times 3} & 0 \\ 0 & 1\end{array}\right)$, where $A_{3 \times 3}$ is an orthogonal $3 \times 3$ matrix, which transforms the surface $z=f(x, y)$ onto another surface $z=F(x, y)$ with horizontal tangent plane. The matrices $Q$ and $A$ represent isometries in $\mathbb{S}_{+}^{3}\left(\mathcal{\kappa}^{-1 / 2}\right)$ and $\mathbb{E}^{3}$, respectively. Since the Gaussian curvature is invariant under isometries there are $a^{\prime}, b^{\prime}$ such that

$$
\kappa_{\mathbb{S}_{+}^{3}\left(\kappa^{-1 / 2}\right)}(\mathbf{r}(a, b))=\frac{\left(1+\kappa a^{\prime 2}+\kappa b^{\prime 2}+\kappa F^{2}\left(a^{\prime}, b^{\prime}\right)\right)^{2}}{\left(1+\kappa F^{2}\left(a^{\prime}, b^{\prime}\right)\right)^{2}} \kappa_{\mathbb{E}^{3}}(a, b, f(a, b))+\kappa .
$$

Therefore, the curvature of a regular surface in $\mathbb{S}_{+}^{3}\left(\kappa^{-1 / 2}\right)$ is not greater than $\kappa$ if and only if the Euclidean curvature of the regular surface in $\mathbb{E}^{3}$, represented by

$$
\mathbf{R}(x, y)=(x, y, f(x, y))
$$

is nonpositive, or equivalently, if and only if $\mathbf{R}(x, y)$ is a saddle surface in $\mathbb{E}^{3}$. But, $\mathbf{R}(x, y)$ is the image of $\mathbf{r}(x, y)$ under the central projection $\varphi$. Hence, Corollary 2.3(ii) completes the proof of Theorem 1.1.

\section{A nonregular nonsaddle surface of nonpositive curvature}

The condition of regularity is necessary for the characterization of saddle surfaces, by means of the intrinsic curvature, to be valid. We present an example of a polyhedral surface in $\mathbb{E}^{3}$ of nonpositive intrinsic curvature, which is not a saddle surface. Applying the inverse of the geodesic mappings id: $\mathbb{H}_{\mathcal{\kappa}}^{3} \rightarrow \mathbb{E}^{3}$ and $\varphi: \mathbb{S}_{+}^{3}\left(\kappa^{-1 / 2}\right) \rightarrow \mathbb{E}^{3}$, we get the corresponding examples in the hyperbolic and spherical space.

Consider the polyhedron $P$ defined by the points $A_{1}(0,0,0), A_{2}(1,0, \varepsilon)$, $A_{3}(0,0,1), A_{4}(0,1, \varepsilon), A_{5}(-1,0, \varepsilon), A_{6}(0,-1, \varepsilon)$, where $\varepsilon$ is any sufficiently small positive number. The bounding curve of $P$ is the polygonal line $A_{2} A_{3} A_{4} A_{5} A_{6} A_{2}$ and the only vertex is the point $A_{1}$. If $\theta(\varepsilon)$ is the total angle of $P$ at the vertex $A_{1}$, then $\lim _{\varepsilon \rightarrow 0} \theta(\varepsilon)=5 \pi / 2>2 \pi$. The intrinsic curvature of $P$ is, by definition, zero everywhere except at the vertex $A_{1}$ where it is equal to $2 \pi-\theta(\varepsilon)$. Therefore, for sufficiently small $\varepsilon>0$, the intrinsic curvature of the polyhedron $P$ is nonpositive. But, on the other hand, for any such $\varepsilon$ the polyhedron $P$ is not a saddle, since we can cut off a crust about the vertex $A_{1}$ by the plane with equation $z=\varepsilon / 2$.

\section{Acknowledgment}

The author is indebted to Igor G. Nikolaev for stimulating discussions on this work. 


\section{References}

[1] Yu. D. Burago and S. Z. Shefel', The geometry of surfaces in Euclidean spaces, Geometry, III, Theory of Surfaces, Encyclopedia Math. Sci., vol. 48, Springer-Verlag, Berlin, 1992, pp. 1-85.

[2] D. E. Kalikakis, On the curvature of nonregular saddle surfaces in the hyperbolic and spherical three-space, Abstr. Appl. Anal. 7 (2002), no. 3, 113-123.

[3] S. Z. Shefel', The two classes of k-dimensional surfaces in n-dimensional Euclidean space, Sibirsk. Mat. Zh. 10 (1969), 459-466, translated from Siberian Math. J. 10 (1969), 328-350.

Dimitrios E. Kalikakis: Department of Mathematics, University of Crete Heraklion, 714-09, Crete, Greece

E-mail address: kalikak@tem.uoc.gr 


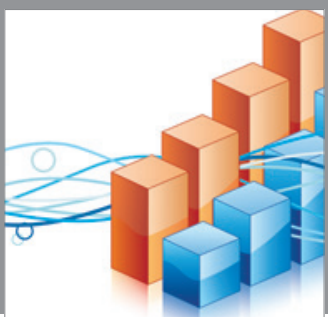

Advances in

Operations Research

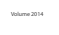

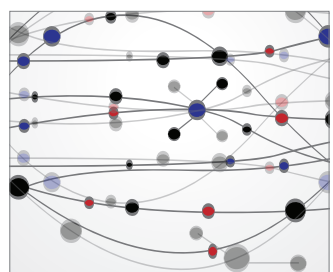

\section{The Scientific} World Journal
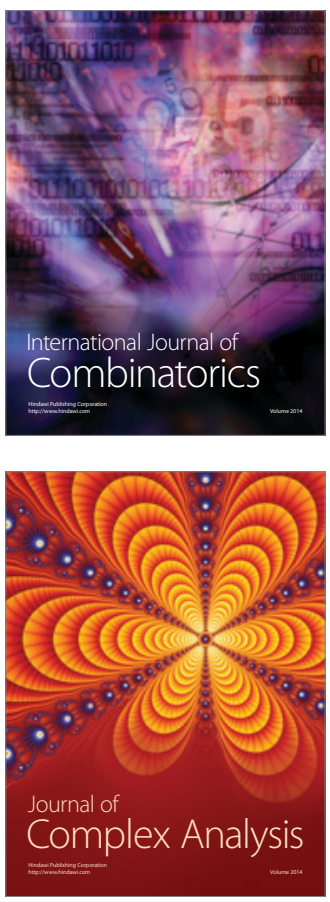

International Journal of

Mathematics and

Mathematical

Sciences
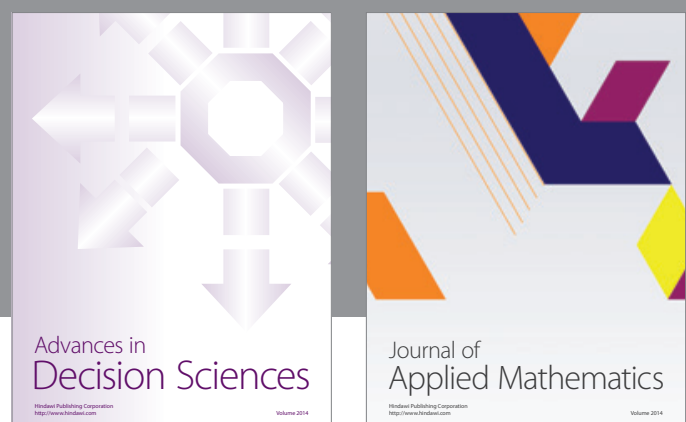

Journal of

Applied Mathematics
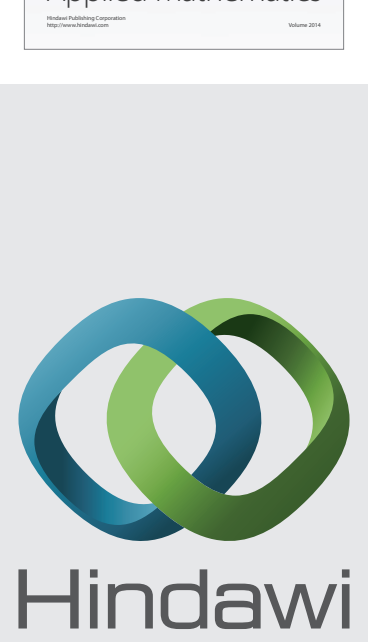

Submit your manuscripts at http://www.hindawi.com
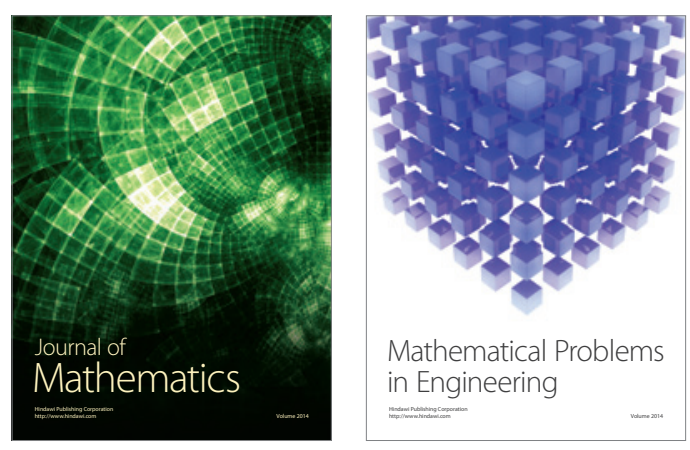

Mathematical Problems in Engineering
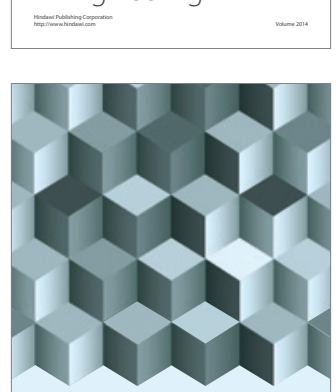

Journal of

Function Spaces
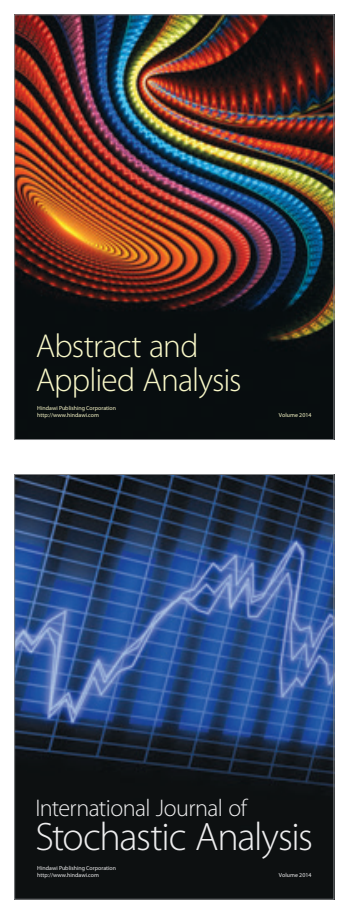

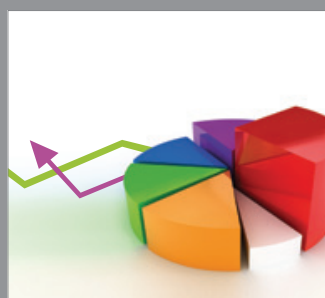

ournal of

Probability and Statistics

Promensencen
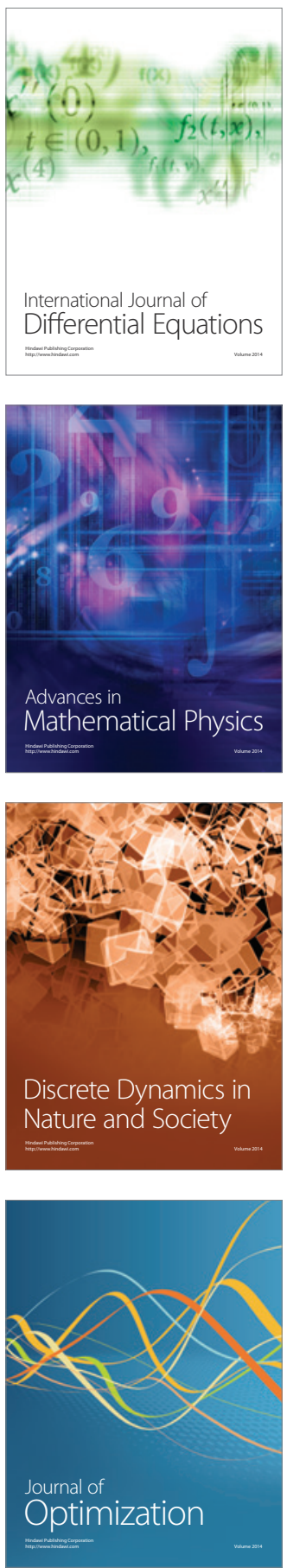